\title{
CARACTERIZACIÓN FÍSICA DE SEMILLAS DE MAÍZ (Zea maǐr) SEMBRADO EN ANDAHUAYLAS PERÚ
}

\author{
PHYSICAL CHARACTERIZATION OF SEED CORN (Zea mays) PLANTED IN \\ ANDAHUAYLAS PERU
}

\author{
${ }^{1}$ Thomas Ancco Vizcarra; ${ }^{1}$ David Juan Ramos Huallpartupa; ${ }^{2}$ Ever Alejo Vivanco Buleje; \\ ${ }^{2}$ Rober Pillaca Ramos; ${ }^{2}$ Heber Alex Andia Salazar
}

\begin{abstract}
RESUMEN
El objetivos del estudio fue determinar las propiedades físicas de semillas de maíz cultivadas en la ciudad de Andahuaylas, se determinaron propiedades físicas: ángulo de reposo, coeficiente de fricción estático, coeficiente de fricción dinámico, esfericidad y área superficial, este último por análisis de imágenes, se consideró como factor en estudio la variedad, morocho (V1), almidón (V2), chullpi (V3) y morado (V4), los datos fueron analizados por análisis estadístico de varianza de solo factor, y sometidos a comparaciones por el método de Duncan; las variedades en estudio mostraron variabilidad en las propiedades físicas, determinándose ángulo de reposo promedio de $26,42^{\circ}$, coeficiente de fricción estático promedio 0,839 , esfericidad promedio de 0,698 , con mayores valores V2, y coeficiente de fricción dinámico promedio 0,286 , con mayor coeficiente dinámico V4; el área superficial promedio fue $1,79 \mathrm{~cm}^{2}$ con mayores áreas V2 y V1. La principal influencia sobre la variabilidad en las propiedades físicas es atribuida a la variedad de maiz.
\end{abstract}

Palabras clave: semillas, ángulo de reposo, esfericidad, coeficiente de fricción.

\section{ABSTRACT}

The objectives of this study were determine the physical properties of seeds of maize cultivated in Andahuaylas city; physical properties were determined: angle of repose, coefficient of static friction, dynamic friction cofficient, sphericity and surface area. This last one for analysis of images; There were considered as factor in study the variety, morocho (V1), starch (V2), chullpi (V3) and purple (V4).Data were analyzed by Statistical analysis of variance of single factor and subjected to comparisons by Duncan method; the varieties under study showed variability in the physical properties, determining angle of repose of $26,42^{\circ}$, static friction coefficient average 0,839 , average sphericity of 0,698 , with major values $\mathrm{V} 2$, and dynamic average friction coefficient 0,286 , with major dynamic coefficient V4. The surface average area was $1,79 \mathrm{~cm}^{2}$ with major areas $\mathrm{V} 2$ and $\mathrm{V} 1$. The principal influence on variability in the physical properties is attributed to the maize variety.

Key words: seeds, angle of repose, sphericity, coefficient of friction.

\section{INTRODUCCIÓN}

En el procesamiento de alimentos, con frecuencia se observa que las características físicas dentro de ellas la forma de las partículas, la capacidad de fluir del material, la fragilidad de los sólidos, el contenido de humedad, la carga estática, influyen en la eficiencia y rendimiento de los equipos de separación, clasificación, reducción de tamaño, transporte; asimismo las partículas con tamaños cercanos a la apertura pueden bloquear el pasaje de las particulas, siendo estas partículas las más difíciles de separar. Considerándolas partículas cercanas a la apertura en el rango $0,75 \mathrm{LA}<\mathrm{dp}<1,5 \mathrm{LA}$, suelen ser las responsables de la pérdida de eficiencia en clasificadores o seleccionadores de alimentos ya que generan obstrucción. Las particulas que son elongadas pueden ser retenidas o atravesar la malla, esto depende del ángulo de aproximación con que lleguen a la superficic de separación (Holdich, 2002). Es por ello que el conocimiento de las propiedades físicas de los granos y semillas de cereales es importante para el diseño y optimización de equipos involucrados con las etapas de procesamiento de productos agrícolas (Mohsenin, 1986; Stroshine y Hamann, 1993; Ospina, 2001; Cetin, 2007; Haddad et al., 1999; Gupta y Das, 2000; Henry et al., 2000; Vursavus y Ozguven, 2004, 2005; Baumler et al., 2006; Altuntas y Yildiz, 2007; Andrejko y Grochowicz, 2007; Dziki, 2007; Correa et al., 2008; Saiedirad el al., 2008; Kilickan y Guner, 2008). Estudios realizados en semillas mostraron que las propiedades físicas son fuertemente influenciadas por el conteniclo de humedad (Mohsenin, 1986). Stroshine y Hamann (1993) y Ospina (2001) mencionan que el conocimiento de las propiedades físicas como el tamaño y forma (esfericidad) de la semilla son necesarios para el diseño de equipos de clasificación y control de calidad; el área proyectada, para el diseño de equipos de transporte y deshidratación; y el coeficiente de

Master of Sciencie en Postcosecha y Marketing. Ingeniero Agroindustrial. Facultad de Ingenieria y Instituto de Investigación y Desarrollo Tecnologico de Recursos Andinos-IIDRA de la Universidad Nacional José Maria Arguedas. Andahuaylas-Perú.

¿Estudiante de Ingenieria Agroindustrial. Asociación de Investigadores Nueva Agroindustria y Transformación Territorial-AINTA de la Universidad Nacional José María Arguedas. Andahuaylas -Peru. 
fricción estático, para el diseño de sistemas de transporte con las que se determina la eficiencia y desgaste de equipos.

Para movilizar granos o semillas en fajas transportadoras o para alimentar materiales mediante tolvas para reducción de tamaño $\mathrm{u}$ otros fines, los materiales granulados o en polvo forman un montículo similar a un cono invertido, donde el ángulo formado por la horizontal y el talud, es definido como el ángulo de reposo, que está influenciado por el tamaño, forma, volumen, densidad, superficie de la semilla, contenido de humedad y orientación (Jha, 1999). El ángulo de reposo para el maíz es $30^{\circ}$ (Ospina, 2001) y es un indicador de la fluidez, necesario para determinar el grado de inclinación de las superficies de transporte y de almacenamiento como tolvas y silos y otros equipos de procesamiento de alimentos.

Los granos o semillas al momento de ser transportados hacia los equipos hacen fricción al entrar en contacto directo con los equipos o partes de los equipos, esta fricción causa generalmente daño mecánico en granos y semillas, generando consecuentemente desgaste de los equipos y sus partes, por tanto el conocimiento del coeficiente de fricción entre el material biológico y la superficie de contacto sirve para conocer y determinar las pérdidas de potencia debida a la fricción en máquinas transportadoras y cosechadoras, es necesario entonces su conocimiento, para asumir criterios de diseño de máquinas y reducir al mínimo daños mecánicos (Lozano, 2002).

La relación entre la fuerza máxima de fricción necesaria para mover las semillas y el peso de éstas, es definida como coeficiente de fricción estático (Altuntas y Yildiz, 2007), en tanto la fuerza para desplazar las semillas sobre la superficie es conocida como el coeficiente de fricción dinámico (Amin et al, 2004). El coeficiente de fricción sobre metal varía de 0,201 a 0,255 dependiendo del contenido de humedad, Ospina (2001) reportó valores de coeficientes de fricción estático de $0,24(9,9 \%), 0,25(12,2 \%)$ y $0,4(13,9 \%)$ para semillas de maíz según su contenido de humedad.

Dentro de las características físicas también es de interés conocer la esfericidad, que es una medida convencional que expresa el grado en que una partícula se aproxima a una esfera. Wentworth (1922) fue el primero en desarrollar una expresión que indica la forma de las partículas, se trata de su Coeficiente de aplastamiento, expresado por ra/R, donde ra es el radio de curvatura de la cara más plana y $\mathrm{R}$ el radio medio. El mismo autor también desarrolló la expresión de redondez ri/ $\mathrm{R}$, donde ri es el radio de curvatura de la arista más aguda. Wadell (1932) propuso la medida de esfericidad verdadera que es igual a s/S donde "S" es el área de la superficie de la partícula y "s" el área de la superficie de una esfera del mismo volumen.

En consecuencia, la esfericidad expresada así, es el área de la partícula por unidad de volumen. Sneed y Folk (1958) revisaron las distintas mediciones de esfericidad y propusieron una nueva: la esfericidad máxima de proyección, Фp (también denominada esfericidad efectiva de sedimentación, que es igual a la razón entre una sección principal de una esfera de igual volumen y el área máxima de proyección de la esfericidad).

La determinación de la redondez puede realizarse directamente sobre la partícula o por comparación con una carta de patrones (Rittenhouse, 1943).

La dificultad de medición de la esfericidad de Wadell (1935) volvió a utilizar la esfericidad definida por Tickell (1947) igual a dc/ Dc, donde dc es el diámetro del círculo de igual área (medido con un planímetro) y Dc es el diámetro menor circunscripto. Sin embargo, Tickell (1947) se inclina por la fórmula de Riley (1941) $\mathrm{Ri}=(\mathrm{di} / \mathrm{Dc}) 1 / 2$, donde di y De son el círculo máximo inscrito y mínimo circunscrito.

El objetivo del estudio fue determinar las propiedades físicas, de semillas de maíz cultivados y comercializados en la región de Apurímac, caracterización que consideramos indispensable para cualquier uso o tratamiento posterior de sus resultados logrados.

\section{MATERIALES Y MÉTODOS}

\section{Obtención y adecuación del material vegetal}

Las semillas de maíz (Zea maiz) en sus cuatro variedades (figuras 1 y 2) se obtuvieron directamente de los productores del Distrito de San Jerónimo Andahuaylas, las mismas que fueron identificadas por el personal especializado del Instituto Nacional de Investigación Agraria INIA establecidos en la ciudad de Andahuaylas ubicada a 2926 m.s.n.m. Para cada prueba las semillas fueron seleccionadas aleatoriamente.

\section{Determinación de humedad}

El contenido de humedad se determinó por triplicado en estufa de aire forzado según el método de ASAE (ASAE, 1999).

\section{Propiedades fisicoquímicas}

Angulo de reposo: se empleó un cilindro hueco de $16,5 \mathrm{~cm}$ de alto y $8,5 \mathrm{~cm}$ de diámetro, abierto en los dos extremos dispuesto perpendicularmente sobre un plano, donde se realizó la lectura del valor (D) y el valor de altura (h) con los datos obtenidos se procedió a determinar el ángulo de reposo con la Ecuación 1. El procedimiento consistió en depositar las semillas de maíz previamente pesadas dentro del cilindro. El llenado se hizo lentamente y sin vibración o compactación para que las semillas de maíz queden lo más suelto posible al interior del cilindro hueco, con las semillas depositadas en el cilindro, se procede a suspender perpendicularmente al plano o base donde la pila se va formando a medida que el material va descargándose, el procedimiento se repite por diez veces, las mediciones de hy $\mathrm{D}$, fueron realizadas con escalímetro y en papel milimetrado.

$$
\theta_{r e p}=\tan ^{-1}\left[\begin{array}{c}
h \\
(D)
\end{array}\right]
$$

Donde:

$\mathrm{h}=$ Altura

$\mathrm{D}=$ Diámetro

Coeficiente de fricción estático: las semillas de maíz en sus cuatro variedades, fueron pesados y depositadas en una caja de madera, de dimensiones de $90 \times 90 \times 90 \mathrm{~mm}$ previamente ubicada sobre la superficie del plano inclinado. Para suprimir 
Ancco, T. et al., Caracterización Física de semillas de maiz (Zea maiz) sembrado en Andahuaylas Perú .

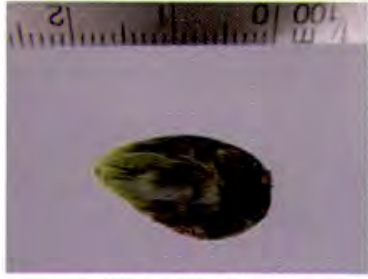

Maíz Chullpi

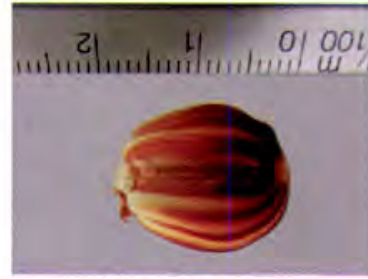

Maíz Morocho

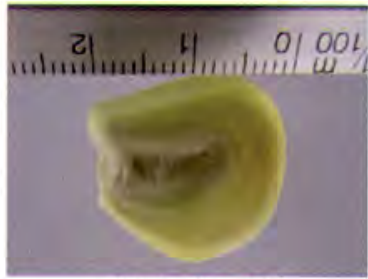

Maíz Almidón

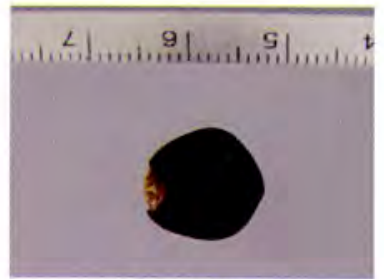

Maíz Morado

Figura 1. Variedades de maíz

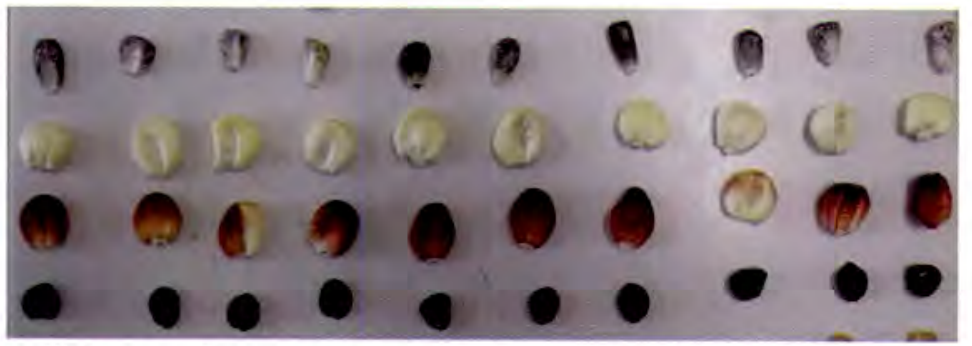

Figura 2. Granos de maíz en orden descendente: Chullpi, Almidón, Morocho y Morado

todo efecto de la base de la caja sobre el coeficiente de fricción, se suspendió $3 \mathrm{~mm}$ quedando solo las semillas de maíz en contacto con la superficie; se suspende progresivamente el plano inclinado hasta lograr romper la máxima fuerza normal de la masa de semillas de maíz con el plano inclinado; logrado el desplazamiento de la masa de maíz, se registra la lectura del ángulo que permitió el desplazamiento de la masa en estudio, valor que se emplea para determinar el coeficiente de fricción estático con la Ecuación 2:

$$
\mu_{s}=\frac{F_{0}}{W} \circ \mu_{s}=\frac{h}{b} * \frac{1}{\tan \theta}
$$

Donde:

$\mu_{f}$ : Coeficiente de fricción estático (adimensional)

Fo: Fuerza máxima requerida para mover la muestra, kilogramos fuerza (kgf).

W: Peso de las semillas más peso de la caja vacía, kilogramos fuerza (kgf).

Coeficiente de fricción dinámico: se determinó en un plano horizontal, para su determinación se pesó la masa de semillas de maíz y se depositó en el soporte de dimensiones $100 \mathrm{~mm} \times 100 \mathrm{~mm} \times 90 \mathrm{~mm}$, previamente dispuesta en posición horizontal en el plano horizontal, de modo que la polea quede libre y pueda deslizarse, y se van depositando masas conocidas hasta que el bloque empieza a deslizarse a velocidad constante, y se registra la masa obtenida. El procedimiento se repitió por diez veces, el coeficiente de fricción dinámico se determina mediante la Ecuación 3. (Altuntas y Yildiz, 2007).

$$
\mu_{c}=\frac{t_{c}}{N}
$$

$\mu_{i}$ : Coeficiente de fricción dinámico (adimensional)

$f:$ Fuerza máxima requerida para mover la muestra, kilogramos fuerza (kgf)

$N$ : Peso de las semillas más peso de la caja vacía, kilogramos fuerza $(\mathrm{kgf})$
Dimensiones y tamaño: se tomaron al azar semillas de maíz, las dimensiones ortogonales: longitud (a), ancho (b), y espesor (c) se determinaron con calibrador (vernier), a partir de ellas, se calculó el diámetro medio geométrico (Dg) y el diámetro medio aritmético (Da), utilizando las siguientes ecuaciones (Altuntas y Yildiz, 2007; Cetin, 2007; Dursun et al., 2007):

$$
\begin{aligned}
& D_{a}=\frac{a \cdot b \cdot c}{3} \\
& D_{g}=(a \cdot b \cdot c)^{1 / 3}
\end{aligned}
$$

Donde:

a: Diámetro Mayor

b: Diámetro Medio

c: Diámetro Inferior

Esfericidad ( $\varphi$ ): Es un criterio definido para determinar la forma de un material biológico, con las dimensiones ortogonales: diámetro mayor (a), diámetro medio (b), y diámetro inferior (c), la determinación de la esfericidad se hizo aplicando la Ecuación 6, según método utilizado por Dursun et al., (2007), Joshi et al., (1993) y Dutta (1988):

$$
\varphi=\frac{(a \cdot b \cdot c)^{1 / 3}}{a}
$$

Area superficial: Se construyó una caja a fin de evitar y eliminar toda posible emisión de sombra al momento de adquirir las imágenes, se empleó también una cámara de 16 mega pixeles, con las que se captaron las imágenes, las que fueron analizadas con el software libre Imagej.

\section{RESULTADOS}

En la Tabla 1, se presenta los resultados de comparación de medias del ángulo de reposo en las variedades de maíz Morocho (V1), Almidón (V2), Chullpi (V3) y Morado (V4), determinándose diferencias estadísticamente significativas al $95 \%$ de confiabilidad.

En la Tabla 2, se presenta la prueba de comparación múltiple de DUNCAN, se observa mayor 
Tabla 1. Análisis de varianza para el ángulo de reposo en cuatro variedades de maíz

\begin{tabular}{lccccc}
\hline Fuente de Variación & S.C. & Gl & C. M. & Razón-F & Valor-P \\
\hline Entre las variedades & 956,265 & 3 & 318,755 & 64,56 & 0,0000 \\
Dentro de la variedad & 177,731 & 36 & 4,936 & & \\
\hline Total (Corr.) & 1134,0 & 39 & & & \\
\hline
\end{tabular}

SC(Suma de cuadrado, $\mathrm{Gl}$ (Grados de libertad), CM(Cuadrado medio de error).

Tabla 2. Comparaciones Múltiples de DUNCAN para el ángulo de reposo en cuatro variedades de maíz

\begin{tabular}{cccc}
\hline Variedad & Observaciones & Media & Significancia \\
\hline V4 & 10 & 22,511 & b \\
V3 & 10 & 23,924 & b \\
V1 & 10 & 24,460 & b \\
V2 & 10 & 34,803 & a
\end{tabular}

ángulo de reposo en $\mathrm{V} 2$, diferenciándose de $\mathrm{V} 1, \mathrm{~V} 3 \mathrm{y} \mathrm{V} 4 \mathrm{en}$ las que no presentaron diferencias estadísticas significativas en las medias comparadas, es decir que el ángulo de reposo es similar para las tres variedades.

En la Tabla 3 se presenta el análisis de varianza para el coeficiente de fricción estático, donde se observa que existen diferencias estadísticas significativas entre las medias del coeficiente de fricción estático de las cuatro variedades de maiz al 95\% de confiabilidad.

Tabla 3. Análisis de varianza para el coeficiente de fricción estático en cuatro variedades de maíz

\begin{tabular}{ccccccc}
\hline Fuente de Variación & S.C. & Gl & C. M. & \multicolumn{2}{c}{ Razón-F Valor-P } \\
\hline Entre las variedades & 0,0000272 & 3 & 0,0000090 & 3,44 & 0,026 \\
Dentro de la variedad & 0,0000951 & 36 & 0,0000026 & & \\
\hline Total (Corr.) & 0,000122 & 39 & & & \\
\hline
\end{tabular}

En la Tabla 4, se presenta la prueba de comparación múltiple de DUNCAN, determinándose mayor coeficiente de fricción estático en V2 a diferencia de $\mathrm{V} 3$, V1 y V4, en las que no se determinó diferencias significativas en las medias comparadas al 95\% de confiabilidad, es decir que el Angulo de reposo es igual para las tres variedades a diferencia de V2, los valores determinados son para un ángulo promedio de $37,5^{\circ}$.

Tabla 4. Comparaciones múltiples de DUNCAN para el coeficiente de fricción estático en cuatro variedades de maíz

\begin{tabular}{cccc}
\hline Variedad & Observaciones & Media & Significancia \\
\hline V4 & 10 & 0,937 & $\mathrm{~b}$ \\
V1 & 10 & 0,937 & $\mathrm{~b}$ \\
V3 & 10 & 0,938 & $\mathrm{ba}$ \\
V2 & 10 & 0,939 & $\mathrm{a}$ \\
\hline
\end{tabular}

En la Tabla 5 se presenta el análisis de varianza para el coeficiente de fricción dinámico, donde se observa que existen diferencias estadísticas altamente significativas entre las medias del coeficiente de fricción dinámico en las cuatro variedades de maíz al 99\% de confiabilidad.

Tabla 5. Análisis de varianza para el coeficiente de fricción dinámico en cuatro variedades de maíz

\begin{tabular}{lllll}
\hline Fuente de Variación S.C. & Gl C. M. & Razón-F & Valor-P \\
\hline Entre las variedades & 0,0124 & 30,0041405 & 10,95 & 0,0000 \\
Dentro de las & 0,0136 & 360,0003781 & & \\
\hline Total (Corr.) & 0,0260 & 39 & & \\
\hline
\end{tabular}

En la Tabla 6, se presenta la prueba de comparación múltiple de DUNCAN, determinándose mayor coeficiente de fricción dinámico en V2 a diferencia de $\mathrm{V} 4$, V1 y V3, en las que no se determinó diferencias significativas en las medias comparadas al $95 \%$ de confiabilidad, es decir que el ángulo de reposo es igual para las tres variedades a diferencia de V2, valores determinados para una fuerza de arrastre promedio de $0,286 \mathrm{~N}$.

Tabla 6. Comparaciones múltiples de DUNCAN para el coeficiente de fricción dinámico en cuatro variedades de maíz

\begin{tabular}{cccc}
\hline Variedad & Observaciones & Media & Significancia \\
\hline V3 & 10 & 0,269 & $\mathrm{~b}$ \\
V1 & 10 & 0,276 & $\mathrm{~b}$ \\
V4 & 10 & 0,283 & $\mathrm{~b}$ \\
V2 & 10 & 0,315 & $\mathrm{a}$ \\
\hline
\end{tabular}

En la Tabla 7 se presenta el análisis de varianza para la esfericidad de los granos de maíz en sus cuatro variedades, donde se observa que existen diferencias estadísticas significativas entre las medias de la esfericidad del maíz al $95 \%$ de confiabilidad.

Tabla 7. Análisis de varianza para la esfericidad de cuatro variedades de maíz

\begin{tabular}{cccccc}
\hline Fuente de Variación & S.C. & Gl & C. M. & Razón-F & Valor-P \\
\hline Entre las variedades & 0,150 & 3 & 0,0502 & 7,98 & 0,0003 \\
Dentro de la variedad & 0,226 & 36 & 0,0062 & & \\
\hline Total (Corr.) & 0,377 & 39 & & & \\
\hline
\end{tabular}

En la Tabla 8, se presenta la prueba de comparación múltiple de DUNCAN, determinándose mayor esfericidad en V2 y V4, a diferencia de V1 y V2 medias comparadas al $95 \%$ de confiabilidad, resaltando que entre V4, V1 y V3 no existen diferencias estadísticas significativas entre sí.

Tabla 8. Comparaciones Múltiples de DUNCAN para la esfericidad en cuatro variedades de maíz

\begin{tabular}{cccc}
\hline Variedad & Observaciones & Media & Significancia \\
\hline V3 & 10 & 0,606 & $\mathrm{c}$ \\
V1 & 10 & 0,685 & $\mathrm{~b}$ \\
V4 & 10 & 0,730 & $\mathrm{ba}$ \\
V2 & 10 & 0,772 & $\mathrm{a}$ \\
\hline
\end{tabular}

En la Tabla 9 se presenta el análisis de varianza para el área superficial de los granos de maiz en sus cuatro variedades, donde se observa que existen diferencias estadísticas significativas entre el área superficial al 95\% de confiabilidad.

Tabla 9. Análisis de varianza para la esfericidad de cuatro variedades de maíz

\begin{tabular}{cccccc}
\hline Fuente de Variación & S.C. & Gl & C. M. & Razón-F & Valor-P \\
\hline Entre las variedades & 18,869 & 3 & 6,289 & 64,06 & 0,0000 \\
Dentro de la variedad & 3,5347 & 36 & 0,098 & & \\
\hline Total (Corr.) & 22,404 & 39 & & & \\
\hline
\end{tabular}

En la Tabla 10, se presenta la prueba de comparación múltiple de DUNCAN, determinándose mayor área superficial en V2 y V1, a diferencia de V3 y V4 medias comparadas al 95\% de confiabilidad, resaltando que entre V1 y V2 no existen diferencias estadísticas significativas entre así como entre V3 y V4. 
Ancco, T. et al., Caracterización Fisica de semillas de maíz (Zea maiz) sembrado en Andahuaylas Perú .

Tabla 10. Comparaciones múltiples de DUNCAN para el área superficial en cuatro variedades de maíz

\begin{tabular}{cccc}
\hline Variedad & Observaciones & Media & Significancia \\
\hline V4 & 10 & 0,995 & b \\
V3 & 10 & 1,247 & b \\
V1 & 10 & 2,349 & a \\
V2 & 10 & 2,595 & a \\
\hline
\end{tabular}

En las tablas 11 al 15 se presenta el resumen de los estadísticos determinados para las cuatro variedades de maíz objeto de estudio, donde observamos los promedio, la desviación standart, el coeficiente de variación, los mínimos y máximos así como el rango para el ángulo de reposo, coeficiente de fricción estático, coeficiente de fricción dinámico obtenido para las cuatro variedades estudiadas.

\section{DISCUSIÓN}

El ángulo de reposo determinado para las cuatro variedades oscila entre 22,5 y 34,8 , superando al valor de $30^{\circ}$ determinado por Ospina (2001), esta variación muy posiblemente sea debido a la orientación de las semillas, tamaño, forma, volumen y densidad como lo menciona Jha (1999), variabilidad que también es atribuida a la forma, volumen, $y$ principalmente a la variedad.

La variación determinada en el coeficiente de fricción estático y dinámico, muy posiblemente sea influenciado por la fuerza máxima de fricción necesaria para mover las semillas y el peso de estas, así como la variedad y densidad propia de cada semilla, dejando manifiesto que para diseñar equipos de transporte, de granos y semillas y aquellas partes que entran en contacto con alimentos son de utilidad (Lozano, 2002) y que su inadecuada elección ocasionarán pérdidas de potencia en las maquinas debida a la fricción ejercida por las semillas en máquinas transportadoras y cosechadoras, los resultados resaltan que la variedad V2 y V3 tienen coeficiente de fricción estático mayor; en tanto V2 y V4 presentaron mayores coeficientes de fricción dinámico; que generarían mayor desgaste en el material de diseño si paralelamente no se considera el ángulo de reposo. Como lo indican Lozano (2002), Altuntas y Yildiz (2007) y Amin et al. (2004) los valores de coeficiente de fricción determinados son próximos a los valores determinados por Ospina (2001), a diferencia de V2 que

Tabla 11. Resumen del análisis estadístico para el Angulo de reposo

\begin{tabular}{cccccccc}
\hline & Recuento & Promedio & Desviación Estándar & Coeficiente de Variación & Mínimo & Máximo & Rango \\
\hline V1 & 10 & 24,460 & 1,090 & $4,459 \%$ & 22,29 & 25,74 & 3,45 \\
V2 & 10 & 34,803 & 4,044 & $11,621 \%$ & 28,72 & 41,01 & 12,29 \\
V3 & 10 & 23,924 & 1,185 & $4,956 \%$ & 22,21 & 25,29 & 3,08 \\
V4 & 10 & 22,511 & 0,891 & $3,958 \%$ & 21,16 & 23,47 & 2,31 \\
Total & 40 & 26,424 & 5,392 & $20,406 \%$ & 21,16 & 41,01 & 19,85 \\
\hline
\end{tabular}

Tabla 12. Resumen del análisis estadístico para el coeficiente de fricción estático

\begin{tabular}{cccccccc}
\hline & Recuento & Promedio & Desviación Estándar & Coeficiente de Variación & Mínimo & Máximo & Rango \\
\hline V1 & 10 & 0,9377 & 0,0017 & $0,189 \%$ & 0,9358 & 0,941 & 0,0052 \\
V2 & 10 & 0,9397 & 0,0019 & $0,206 \%$ & 0,9358 & 0,9423 & 0,0065 \\
V3 & 10 & 0,9382 & 0,0013 & $0,144 \%$ & 0,9358 & 0,9394 & 0,0036 \\
V4 & 10 & 0,9376 & 0,0013 & $0,144 \%$ & 0,9358 & 0,9389 & 0,0031 \\
Total & 40 & 0,9383 & 0,0017 & $0,188 \%$ & 0,9358 & 0,9423 & 0,0065 \\
\hline
\end{tabular}

Tabla 13. Resumen del análisis estadístico para el coeficiente de fricción dinámico

\begin{tabular}{cccccccc}
\hline & Recuento & Promedio & Desviación Estándar & Coeficiente de Variación & Mínimo & Máximo & Rango \\
V1 & 10 & 0,276 & 0,012 & $4,415 \%$ & 0,249 & 0,2901 & 0,041 \\
V2 & 10 & 0,315 & 0,013 & $4,175 \%$ & 0,2817 & 0,3277 & 0,046 \\
V3 & 10 & 0,269 & 0,023 & $8,807 \%$ & 0,2328 & 0,2987 & 0,066 \\
V4 & 10 & 0,283 & 0,025 & $8,853 \%$ & 0,2382 & 0,3135 & 0,075 \\
Total & 40 & 0,286 & 0,025 & $9,031 \%$ & 0,2328 & 0,3277 & 0,0949 \\
\hline
\end{tabular}

Tabla 14. Resumen del análisis estadístico para la esfericidad

\begin{tabular}{cccccccc}
\hline & Recuento & Promedio & Desviación Estándar & Coeficiente de Variación & Mínimo & Máximo & Rango \\
\hline V1 & 10 & 0,685 & 0,074 & $10,884 \%$ & 0,59 & 0,857 & 0,267 \\
V2 & 10 & 0,772 & 0,088 & $11,401 \%$ & 0,615 & 0,883 & 0,268 \\
V3 & 10 & 0,606 & 0,076 & $12,615 \%$ & 0,498 & 0,738 & 0,240 \\
V4 & 10 & 0,730 & 0,077 & $10,631 \%$ & 0,620 & 0,860 & 0,240 \\
\hline Total & 40 & 0,698 & 0,098 & $14,085 \%$ & 0,498 & 0,883 & 0,385 \\
\hline
\end{tabular}

Tabla 15. Resumen del análisis estadístico para área superficial

\begin{tabular}{cccccccc}
\hline & Recuento & Promedio & Desviación Estándar & Coeficiente de Variación & Mínimo & Máximo & Rango \\
\hline V1 & 10 & 2,349 & 0,325 & $13,847 \%$ & 1,767 & 2,922 & 1,155 \\
V2 & 10 & 2,595 & 0,324 & $12,516 \%$ & 2,138 & 3,142 & 1,004 \\
V3 & 10 & 1,247 & 0,264 & $21,213 \%$ & 1,025 & 1,933 & 0,908 \\
V4 & 10 & 0,995 & 0,333 & $33,516 \%$ & 0,665 & 1,878 & 1,213 \\
Total & 40 & 1,796 & 0,757 & $42,180 \%$ & 0,665 & 3,142 & 2,477 \\
\hline
\end{tabular}


presento 0,315 , variación que es atribuido específicamente la variedad de maiz.

La esfericidad y área superficial permite caracterizar y determinar la forma que toma la partícula, que facilita la adecuada elección y decisión al momento de elegir un equipo o máquina de proceso, como los seleccionadores en control de calidad de granos y productos procesado como indican Mohsenin (1986), Stroshine y Hamann (1993), Ospina (2001); asimismo su conocimiento permite determinar el adecuado caudal másico de alimentación, y el interés en mejorar la eficiencia en los equipos de procesamiento de alimentos (Holdich, 2002).

\section{CONCLUSIONES}

Delos resultados efectuados en las la propiedades físicas determinadas, la variedad Almidón (V2) representa mayor desgaste en equipos de transporte y partes en contacto con alimentos.

Los valores determinados de esfericidad en V2 y V1, V3 y V4, permiten definir a priori la eficiencia en el desempeño de los equipos de tamizado de granos de maíz, cuyos valores facilitan la determinación del caudal másico de la alimentación en los equipos de transporte, molienda y otros relacionados al manejo de granos o semillas de maíz según la variedad.

\section{AGRADECIMIENTOS}

Los autores agradecen al Instituto de Investigación y Desarrollo Tecnológico de Recursos Andinos IIDRA adscrito a la Escuela Profesional de Ingeniería Agroindustrial, Universidad Nacional José María Arguedas.

\section{REFERENCIAS BIBLIOGRÁFICAS}

Altuntas, E. y Yildiz, M. (2007). Effect of moisture content on some physical and mechanical properties of faba bean (Vicia faba L.) grains. Journal of Food Engineering 78(1):174-183.

Amin M.N., Hossain, M.A. and Roy, K.C. (2004). Effects of moisture content on some physical properties of lentil seeds. Journal of Food Engineering 65(1): 83-87.

Andrejko, D. and Grochowicz, J. (2007). Effect of the moisture content on compression energy and strength characteristic of lupine briquettes. Journal of Food Engineering 83(1): 116-120.

ASAE (1999). ASAE Standards Engineering Practices Data. 46th Ed. St. Joseph, MI, USA, American Society of Agricultural Engineers, pp. 567, 512-528.

Baumler, E., Cuniberti, A., Nolasco, S. and Riccobene, I. (2006). Moisture dependent physical and compression properties of safflower seed. Journal of Food Engineering 72(2): 134-140.

Cetin, M. (2007). Physical properties of barbunia bean (Phaseolus vulgaris L. cv. 'Barbunia') seed. Journal of Food Engineering 80: 353-358.

Correa, P.C., Resende, O., Menezes, R., Jarén, C. y Arazuri, S. (2008). Resistance of edible beans to compression. Journal of Food Engineering 86(2):172-177.
Dursun, I., Tugrul, K.M. and Dursun, E. (2007). Some physical properties of sugar beet seed. Journal of Stored Products Research 43(2): 149-155.

Dutta, S.K., Nema, V.K. and Bhardwaj, R.K. (1988). Physical properties of gram. Journal of Agricultural Engineering Research 39(4):259-268.

Dziki, D. (2007). The crushing of wheat kernels and its consequence on the grinding process. Powder Technology 185(2): 181186.

Gupta, R.K. and Das, S.K. (2000). Fracture resistence of sunflower and kernel to compressive loading. Journal of Food Engineering 46:1-8.

Haddad, Y., Mabille, F., Mermet, A., Abecassis, J. and Benet, J.C. (1999). Rheological properties of wheat endosperm with a view on grinding behaviour. Powder Technology 105(13):89-94.

Henry, Z.A., Su, B. and Zhang, H. (2000). Resistance of soya beans to compression. Journal of Agricultural Engineering Research 76(2): 175-181.

Holdich, R.G. (2002). Fundamentals of Particle Tecbnology, Midland Information Technology and Publishing, United Kingdom.

Jha, S.N. (1999). Physical and hygroscopic properties makhana. Journal of Agricultural Engineering Research 72(2):145-150.

Joshi, D.C., Das, S.K. and Mukherji, R.K. (1993). Physical properties of pumpkin seeds. Journal of Agricultural Engineering Research 54:219-229.

Kilickan, A. and Guner, M. (2008). Physical properties and mechanical behavior of olive fruits (Olea europaea L.) under compression loading. Journal of Food Engineering 87(2): 222-228.

Lozano, O. F., (2002) Establecimiento y caracterización de los parámetros de diseño y construcción de máquinas cosechadoras de papa para condiciones colombianas. Bogotá: CORPOICA

Mohsenin, N. (1986). Physical properties of food and agricultural materials, a teacbing manual. Gordon and Breach Science Publishers, New York. $147 \mathrm{p}$.

Ospina, J.E. (2001). Características físico mecánicas y análisis de calidad de granos. Universidad Nacional de Colombia. Departamento de Ingeniería Agrícola, Bogotá. 225 p.

Riley, N. A., (1941), Projection sphericity: Journal Sedimentary Petrology, v. 11, p. 94-97.

Rittenhouse, G. (1943). A visual method of estimating two dimensional sphericity, Journal of Sedimentary Petrology 13: 79-81.

Saiedirad M.H., Tabatabaeefar, A., Borghei, A., Mirsalehi, M., Badii, F., Ghasemi Varnamkhasti M. (2008). Effects of moisture content, seed size, loading rate and seed orientation on force and energy required for fracturing cumin seed (Cuminum cyminum Linn) under quasi-static loading. Journal of Food Engineering 86(4): 565-572.

Sneed E.D. y Folk, R.L. (1958). Pebbles in the lower Colorado River, Texas, a study in particle morphogenesis, Journal of Geology 66(2):114-150

Stroshine, R. and Hamann, D. (1993). Physical properties of agricultural materials and food products. Department of 


\section{Ciencia \& Desarrollo}

Ancco, T. et al., Caracterización Física de semillas de maiz (Zea maiz) sembrado en Andahuaylas Perú .

Food Science, North California State University, LA, California. pp. 82-112

Tickell, F.G. (1947). The examination of fragmental rocks, $3^{2}$ edición. Stanford University Press. 154 pp

Vursavus, K. and Ozguven, F. (2004). Mechanical behaviour of apricot pit under compression loading. Journal of Food Engineering 65(2): 255-261.

Vursavus, K. and Ozguven, F. (2005). Fracture resistance of pine nut to compressive loading. Biosystems Engineering 90(2): 185-191.

Wadell, H. (1932). Volume, shape, and roundness of rock particles, Journal of Geology 40: 443-451.

Wadell, H. (1935). Volume, shape and roundness of quartz particles, Journal of Geology 43: 250-280.

Wentworth, C.K. (1922). A scale of grade and class terms

for clastic sediments. Journ Geol, 30, 377-392

\section{Correspondencia:}

Thomas Ancco Vizcarra: thoanviz@gmail.com

Fecha de Recepción: 04/10/2014

Fecha de Aceptación: 04/12/2014 